\title{
Le modèle allemand
}

\section{Nando Mäsch}

\section{OpenEdition}

Journals

Édition électronique

URL : http://journals.openedition.org/ries/3953

DOI : 10.4000/ries.3953

ISSN : 2261-4265

\section{Éditeur}

Centre international d'études pédagogiques

\section{Édition imprimée}

Date de publication : 7 septembre 1995

Pagination : 45-58

ISSN : 1254-4590

\section{Référence électronique}

Nando Mäsch, «Le modèle allemand », Revue internationale d'éducation de Sèvres [En ligne], 07 | 1995, mis en ligne le 02 octobre 2014, consulté le 21 avril 2019. URL : http://journals.openedition.org/ ries/3953 ; DOl : 10.4000/ries.3953

Ce document a été généré automatiquement le 21 avril 2019

(c) Tous droits réservés 


\title{
Le modèle allemand
}

\author{
Nando Mäsch
}

\section{Genèse et développement actuel}

1 Lors de la première conférence d'experts organisée conjointement par la Commission de la communauté européenne et le Conseil de l'Europe pour la coopération culturelle en mai 1990 à Namur, le type d'éducation bilingue développé en Allemagne depuis le deuxième moitié des années soixante a été déclaré exemplaire. Le concept, en tant que cycle de formation destiné à une population d'élèves autochtones, peut être réalisé partout, même dans les petites communes; il a convaincu les experts parce qu'il représente la politique linguistique de l'Union européenne et du Conseil de l'Europe.

2 C'est depuis cette conférence qu'on parle du « modèle allemand » de l'éducation bilingue. Celle-ci se réalise dans des filières de formation bilingue qui existent en Allemagne sous la forme de Bilingualer Zug (section bilingue) dans les Gymnasien.

3 Le Gymnasium allemand est une entité scolaire de neuf ans correspondant à l'enseignement secondaire français (collège et lycée). Il part de la classe 5 (CM2) et aboutit à l'Abitur, équivalent allemand du baccalauréat français, à la fin de la classe 13.

4 Dans les années quatre-vingt-dix, le principe de l'éducation bilingue s'est répandu dans d'autres types d'établissement.

5 À l'heure actuelle, plus de deux cents établissements scolaires proposent en Allemagne l'éducation bilingue dans une filière du type section bilingue, dont environ cent trente anglo-allemandes, soixante franco-allemandes et très peu de sections visant d'autres langues cibles : le néerlandais, l'espagnol, l'italien, le russe.

6 Les établissements scolaires, qui offrent une section bilingue à côté de leur «section normale», se trouvent dans presque tous les Länder fédéraux. Ils sont situés pour la plupart en Rhénanie-du-Nord-Westphalie, là où l'on dispose de la plus grande expérience. Ceci s'est reflété en 1988 dans les premières Recommandations concernant les matières nonlinguistiques (géographie, instruction civique, histoire), émises par le Kulturminister. Ces premières recommandations qui complètent les programmes sont destinées aux sections 
franco-allemandes. La Fédération des lycées à section bilingue franco-allemande en Allemagne, existant déjà depuis 1975, avait réuni et analysé les expériences de manière qu'elles puissent servir de base pour les décisions fondamentales à prendre.

\section{Le concept européen de l'éducation bilingue}

7 Les sections bilingues sont des « filles » du traité de coopération franco-allemande signé par Charles de Gaulle et Konrad Adenauer en 1963. En Allemagne, l'éducation bilingue est donc née de la volonté de réconciliation. L'idée des premières années était de parvenir à une meilleure compréhension du voisin européen en passant par la compréhension de la langue. Après l'hostilité dite héréditaire, on voulait alors arriver au partenariat.

Jusque vers la fin des années quatre-vingt, les sections bilingues étaient - sauf trois exceptions - toutes des sections franco-allemandes.

C'est en partant de l'idée du partenariat que vivent les sections bilingues, non seulement de par leur histoire mais aussi en tant que concept pédagogique bien réfléchi. L'éducation bilingue veut faire passer la langue du voisin comme étant la «langue du partenaire »; ceci dépasse de loin l'enseignement d'une langue comme lingua franca, c'est-à-dire comme moyen de communication d'une valeur neutre. spécifiques du cursus bilingue. ${ }^{1}$

On doit, cependant, constater que les représentants des sections bilingues angloallemandes ne sont pas tous d'accord sur ce point.

À l'exception de cette minorité, la majorité des représentants de toutes les autres langues cibles est convaincue que l'Europe doit se faire sur la base d'une politique linguistique égalitaire. C'est pour cette raison que les sections bilingues mettent en pratique le concept de partenariat comme idée européenne tournée vers l'avenir. Elles se veulent des " écoles pour l'Europe » et la formation que l'on y pratique veut être une formation à dimension européenne. Elles sont consciemment engagées dans la politique linguistique officielle de l'Union européenne qui est celle de la diversification.

13 L'idée de partenariat doit devenir de plus en plus le principe de base des relations intérieures dans l'Europe en croissance. Une communauté - et c'est ce que voulait être l'ancienne Communauté européenne et veut être l'actuelle Union européenne - ne supporte aucune suprématie, notamment aucune domination linguistique.

L'idée d'une seule « langue de référence " pour l'Europe entière ne correspond pas à une telle politique linguistique. Le terme allemand de Leitsprache est révélateur : cette langue domine per definitionem, elle est hégémonique. Comme la pensée est guidée par la langue, une "langue de référence" non seulement oppresse les autres langues, mais elle fait également obstacle aux autres civilisations et met ainsi leur existence en danger. L'Europe ne conserve pas son identité en passant par une seule «langue de référence " mais par la diversification linguistique.

\section{Le concept pédagogique}

En parlant d'éducation bilingue du "modèle allemand", il est utile de dissiper un malentendu possible : ce ne sont pas les élèves commençant leur carrière au secondaire 
qui sont bilingues, mais c'est la formation, elle, qui se fait en deux langues, en langue première (maternelle) et en langue étrangère. Car l'éducation bilingue du «modèle allemand » est en premier lieu destinée à la population scolaire allemande.

Il pourrait y avoir un second malentendu : le «modèle allemand » ne se veut pas l'unique type d'éducation bilingue. Principalement il vise, en effet, la population scolaire allemande, et il est capable d'accueillir un nombre limité de locuteurs natifs de la langue cible. Pour une population binationale, multinationale ou migrante, il faut prévoir d'autres types d'éducation spécialisés englobant deux ou plusieurs langues et cultures d'origine. En Europe, on en aura de plus en plus besoin.

L'idée maîtresse de la formation bilingue scolaire est la mise en pratique de la langue cible dans des disciplines non linguistiques. Au contraire des programmes d'immersion canadiens et autres, l'enseignement s'étend aussi à la première langue : il est donc bilingue. C'est ainsi qu'en Allemagne le terme bilinguales Sachfach a été créé. Il comporte en lui-même sa définition : il s'agit d'une discipline (Fach) du domaine des objets concrets (Sache) qui est enseignée en deux langues. En français, le terme "discipline bilingue » pourrait rendre service ${ }^{2}$.

L'enseignement des disciplines bilingues est dispensé sur la base d'un enseignement de la langue cible en première langue à partir de la classe 5 . Les élèves ont alors dix ans. En Allemagne, il va sans dire qu'une section bilingue propose l'enseignement d'au moins une deuxième langue étrangère. Au Gymnasium, type d'établissement qui sélectionne les élèves et qui est fréquenté par près de $40 \%$ d'une tranche d'âge (près de $30 \%$ passent l' Abitur qui correspond au baccalauréat général français) ${ }^{3}$, deux langues étrangères sont obligatoires. Environ 80 \% des élèves dans les sections bilingues choisissent une troisième langue étrangère, souvent en plus du latin. Ainsi, le plurilinguisme (l'individu polyglotte), tant sollicité pour l'Europe, est déjà réalisé par les élèves des sections bilingues du «modèle allemand».

\section{Les objectifs}

19 L'éducation bilingue développe une compétence culturelle et linguistique à forte tendance biculturelle et interculturelle. Le but est clair, mais il faut juger les résultats avec objectivité: pour une population scolaire autochtone il ne peut s'agir que de compétences qui s'approchent de celles d'un locuteur natif ou d'un individu biculturel par naissance. Cependant, les compétences atteintes sont d'un très haut niveau, elles sont plus vastes et plus approfondies que celles auxquelles les élèves peuvent arriver en passant par les cursus normaux.

Un arrêté ministériel du Land Rhénanie-du-Nord-Westphalie ${ }^{4}$ définit les objectifs comme suit : l'éducation bilingue vise une compétence linguistique à forte tendance bilingue dans deux langues véhiculaires, la langue cible (LV2) et la langue maternelle (LV1). Elle permet aux élèves à la fin de leur scolarité (Abitur) de communiquer oralement et par écrit sur des aspects importants de la société, de l'État, de l'économie et de la culture, tout en exprimant leurs propres opinions de façon adéquate.

Les objectifs linguistiques impliquent les compétences biculturelles et interculturelles. 


\section{Les enseignants}

Pour mettre en pratique ces concepts et pour atteindre ces buts, il faut des enseignants qualifiés. Il est évident que les sections bilingues doivent pouvoir disposer d'enseignants qui maîtrisent la langue cible à un niveau égal d'un locuteur natif ou qui en soit très proche. Quant à l'aspect de la compétence culturelle, l'enseignant parfait est évidemment le locuteur natif qui porte en lui-même l'identité culturelle qu'il doit transmettre. Il faut ajouter deux précisions :

- l'enseignant parfait, le locuteur natif qui est diplômé pour les deux degrés du secondaire allemand, n'est pas toujours disponible. Il faut donc avoir recours à des enseignants allemands présents dans l'établissement scolaire. Heureusement il n'est pas rare qu'il y ait parmi eux des professeurs dont la compétence s'approche de celle du type idéal ;

- la deuxième précision porte sur les enseignants des disciplines bilingues; il est judicieux d'insister sur leurs compétences, car les conditions diffèrent beaucoup entre les pays, par exemple entre l'Allemagne et la France.

Voici les quatre compétences maîtresses :

- la compétence disciplinaire ${ }^{5}$;

- la compétence linguistique pour la langue cible ;

- la compétence linguistico-didactique ;

- la compétence linguistique de la LV1 des élèves.

Les deux premières sont des compétences indispensables. La troisième et la quatrième dans l'ordre - sont néanmoins d'une importance décisive pour le succès de l'éducation bilingue. Les vingt-cinq années d'expériences en Allemagne ont prouvé qu'il ne faut pas sous-estimer les deux dernières compétences.

En Allemagne, tous les enseignants du secondaire sont, sauf exception, bivalents, c'est-àdire diplômés dans deux disciplines scolaires. Parmi les professeurs de langue, il y a donc quantité de collègues qualifiés pour enseigner par exemple l'anglais et la géographie ou le français et l'histoire ou le néerlandais et l'instruction civique, pour citer les trois disciplines bilingues les plus importantes et les plus fréquentes.

Au début, dans les années soixante-dix, on a constaté en Allemagne que l'enseignement bilingue reste déficitaire si, chez l'enseignant, l'une des quatre compétences n'est pas suffisamment développée, par exemple la compétence en langue cible chez le professeur germanophone de la discipline bilingue, ou la compétence disciplinaire chez le professeur francophone de langue, ou la compétence linguistico-didactique chez le professeur francophone d'histoire-géographie. Il a fallu longtemps pour cerner l'importance de ce second point.

Or, c'est avant tout la compétence complète dans la matière qui est indispensable. Dans le désir de perfectionner la compétence linguistique des élèves dans une section bilingue, il ne faut jamais perdre de vue que la partie bilingue est un additum. Par conséquent, elle ne doit en aucun cas prendre le pas sur le contenu disciplinaire. Le professeur de langue qui n'est pas qualifié dans la discipline n'est pas apte à conduire un enseignement bilingue : une source historique n'est pas une "leçon ", son approche didactique n'est pas identique à celle d'un cours de langue. Les Studienseminare en Allemagne et les institutions homologues en France, les IUFM, insistent à juste titre sur ces différences. 
Quant à l'enseignant de la discipline bilingue, personne ne niera l'importance de la langue cible, qui est la langue véhiculaire quasi permanente du cours. Sa compétence doit rapprocher de celle du locuteur natif. Il va de soi que le professeur francophone qui enseigne, par exemple, la Troisième République dans une section bilingue francoallemande en Allemagne, est particulièrement qualifié pour faire ce travail. Il est rare que l'enseignant qui n'est pas locuteur natif connaisse déjà, au début de son emploi dans la section bilingue, le discours et tous les termes du langage spécifique à la matière. Sa compétence linguistique et sa volonté d'apprendre doivent cependant être telles qu'il soit capable de se perfectionner jusqu'au niveau adéquat.

La troisième compétence est, de fait, indispensable elle aussi. Le professeur qui enseigne une discipline spécifique doit savoir soutenir l'enseignement de la langue étrangère, plus précisément il doit maitriser les procédés et moyens didactiques de l'enseignement des langues étrangères. Sinon, la longue expérience allemande a prouvé que l'enseignement disciplinaire reste inefficace. Que les amateurs des méthodes traditionnelles ne se trompent pas: le cours magistral qui «s'apprend»- la langue avec le contenu - ne correspond plus aux exigences de l'éducation. L'autonomie de l'élève porte, bien évidemment, sur la matière à étudier. Mais "étudier " une matière implique l'emploi réfléchi de la langue.

L'un est intimement lié à l'autre. Ce qui est déjà vrai pour l'enseignement spécifique en langue maternelle l'est d'autant plus en langue étrangère. Là, un professeur qui n'est ni sensible aux problèmes linguistiques posés par la matière ni compétent pour les résoudre, est mal préparé à sa tâche.

En Allemagne, ce problème ne se pose pas. Si, dans un autre contexte national, le manque d'enseignants bivalents est chronique, il est capital de prévoir une formation supplémentaire portant sur les méthodes, les procédés et les savoir-faire en didactique des langues étrangères. Il faut veiller à ce que cette double compétence demandée ne mène pas à une « double demi-compétence ». Analogue au bilinguisme à atteindre par les élèves, la deuxième compétence de l'enseignant est un additum qui est digne d'un prestige complémentaire - et d'avantages sous forme, par exemple, de promotion ou de réduction du nombre de cours obligatoire.

Le professeur étranger une fois intégré dans le système éducatif doit disposer des compétences linguistiques nécessaires pour pouvoir y participer en toute liberté et mobilité sans être gêné par une compétence insuffisante en allemand. Quant à son travail dans les cours spécifiques en langue étrangère, il faut être conscient du concept qui veut que l'éducation soit bilingue, qu'elle implique donc aussi la formation dans la LV1 de l'élève. C'est un avantage indiscutable que l'enseignant soit parfaitement bilingue, mais ce n'est pas indispensable. Il suffit qu'il dispose des moyens linguistiques suffisants pour pouvoir faire passer les termes et le discours spécifique de sa matière dans la LV1 des élèves. Ces moyens doivent aussi être tels qu'ils puissent le mettre à même de développer ses connaissances et ses compétences.

\section{La structure}

Pour un pays comme la République fédérale d'Allemagne où l'autonomie culturelle des Länder fédéraux implique celle de l'éducation, on s'attend à ce que les structures des cursus bilingues diffèrent d'un Land à l'autre et d'un type d'établissement à l'autre. Mais 
en ce qui concerne les sections bilingues des Gymnasien le développement a été uniforme : on peut, à juste titre, parler d'un seul "modèle allemand" qui se réalise sous deux variantes. La description suivante est adoptée par le Land le plus peuplé, la Rhénanie-duNord- Westphalie. Ce Land est, de surcroît, celui avec le plus grand nombre d'établissements scolaires à section bilingue. C'est la structure la plus répandue dans tous les Länder, à l'exception de la Rhénanie-Palatinat.

La structure s'est développée dans les années soixante-dix. Elle a été présentée, pour la première fois en 1985, par l'auteur de cet article ${ }^{6}$ qui était alors proviseur d'un Gymnasium à section bilingue franco-allemande. Elle est née et elle a été développée dans la pratique, de sorte qu'elle s'est révélée opérationnelle et efficace. Jusqu'à ce jour on n'a pas vu la nécessité de la modifier.

$\mathrm{Au}$ cours du cycle «probatoire » (classes 5 et 6 ), la langue cible est la première langue étrangère. Son enseignement est renforcé de deux périodes supplémentaires par semaine (sept au lieu de normalement cinq).

À partir de la classe 7, les disciplines concernées - géographie, instruction civique (classe 8) et histoire (classe 9) - sont progressivement enseignées en langue étrangère. Ces disciplines non linguistiques forment les éléments caractéristiques de la section bilingue. Dans leur première année la géographie et l'instruction civique bénéficient d'une période hebdomadaire supplémentaire de façon à compenser la progression plus lente dans la matière, conséquence d'une compétence linguistique encore réduite. Lors du premier degré de l'enseignement secondaire (classes 5 à 10), la somme totale des cours dispensés en langue cible représente environ un quart du total hebdomadaire des périodes.

Au second degré (classes 11 à 13 du Gymnasium) les élèves qui continuent la formation bilingue suivent dix périodes de cours de langue plus trois dans l'une des disciplines bilingues selon leur choix (histoire ou géographie ou, depuis 1995, sciences sociales).

À l'Abitur, le candidat «bilingue » se présente à des épreuves portant sur la langue cible et à des épreuves sur la discipline de la langue cible : langue cible à l'écrit et, le cas échéant à l'oral ; discipline bilingue en langue étrangère à l'écrit ou à l'oral.

Dans la section bilingue franco-allemande, le candidat reçu obtiendra la «mention bilingue franco-allemande " sur son diplôme de l'Abitur. La mention est le fruit d'un accord entre la France et l'Allemagne. C'est un atout d'une valeur précieuse pour l'entrée et l'avancement dans la vie professionnelle. Pour ceux qui s'engagent dans les études en France, c'est une clé qui dispense du test linguistique pour accéder à l'université.

\section{Avantages et inconvénients du « modèle allemand »}

Dans ces structures relativement homogènes, deux groupes se différencient au point de vue du recrutement des enseignants de sorte qu'on peut parler de deux variantes $d u$ « modèle allemand ».

La " variante additive » n'est pratiquée qu'en Rhénanie-Palatinat ${ }^{7}$. Obéissant à la volonté d'engager le plus possible des locuteurs natifs, une partie des cours renforcés de langue et des disciplines bilingues est dispensée par un locuteur natif. L'autre partie par un enseignant germanophone. 
41 Avantage : dans les disciplines bilingues, la répartition des cours entre langue étrangère et allemand garantit des modèles linguistiques et culturels authentiques dans les deux langues. La coopération indispensable entre les deux enseignants peut être fructueuse. Inconvénients: l'engagement de deux professeurs pour le même enseignement disciplinaire a pour conséquence une répartition des contenus et une séparation du travail linguistique. Cependant, l'apprentissage de la matière et celui de la langue sont intimement liés. Les deux professeurs sont obligés de pratiquer une coopération étroite. Ceci est facile dans son principe mais difficile à réaliser. De plus, la distribution de l'enseignement sur deux professeurs rend difficile l'évaluation des résultats des élèves au travers d'une seule note.

La « variante intégrative » est pratiquée par tous les autres Länder, y compris ceux qui se sont décidés dernièrement à offrir une éducation bilingue : le Bade-Wurtemberg et les nouveaux Länder. Dans le cas idéal, les cours sont faits d'une manière intégrative par des locuteurs natifs, sans séparer contenus et enseignement linguistique.

44 Avantage : le contenu des disciplines bilingues n'est pas réparti sur deux enseignants. Ainsi, l'interdépendance entre l'apprentissage de la matière et celui de la langue est-elle respectée. C'est l'aspect didactique le plus important. L'interaction entre les deux champs d'apprentissage est possible à tout moment donné.

Inconvénient: comme il n'y a pas encore assez d'enseignants correspondant aux exigences, il peut arriver que les élèves manquent de modèles de langue et de culture authentiques.

Par principe, aussi bien pour l'une que pour l'autre variante du modèle, les cours dans les disciplines spécifiques ne sont pas des cours de langue bien qu'ils s'inscrivent sciemment sur une partie des outils méthodologiques acquis au cours de langue.

\section{Le programme}

47 Comme le «modèle allemand » s'adresse principalement à la population allemande, il est logique que le cursus bilingue suive le programme du Land fédéral. Les objectifs d'une compétence bilingue liée à une compétence biculturelle oblige à introduire partout où c'est possible la perspective du "partenaire » et à la comparer à celle de la première culture de l'élève. C'est un principe de l'éducation bilingue selon le « modèle allemand ». En dehors des points obligatoires, les programmes allemands laissent assez de liberté pour y introduire la double perspective souhaitée.

Suivre ce principe ne dispense pas néanmoins de traiter les données spécifiques ayant une influence sur la culture du «partenaire » qui n'ont pas de correspondance dans sa propre civilisation; il est inadmissible de renoncer, par exemple, à la première et la seconde unification de l'Allemagne pour une section européenne du type allemand, en France ou au laïcisme français dans une section bilingue franco-allemande, en Allemagne.

Si l'on examine, à titre d'exemple, le programme du premier semestre de la classe 9 en section bilingue franco-allemande selon les nouvelles recommandations du Land de Rhénanie-du-Nord-Westphalie pour l'histoire comme discipline bilingue ${ }^{8}$, on constate que des sujets d'enseignement d'une importance historique universelle n'ont pas été laissés à l'écart'. 

arriver est l'abandon du cours magistral et la pratique du cours interactif. C'est une stratégie indispensable pour promouvoir l'expression orale des élèves dans la langue cible. leur expression. L'interdépendance de l'apprentissage de la langue et des contenus prime dans les disciplines bilingues; la grammaire s'apprend en fonction de la volonté d'expression et non comme un but en soi.

manifeste dans la compétence à connaître et à choisir les méthodes et les procédés appropriés. Elle implique une compétence sociale qui choisit la forme (sociale) du travail la plus propice à l'approche d'un problème, comme par exemple le travail de groupe. La méthodologie spécifique de la discipline est thématisée et enseignée dans les cours. surtout dans les petites classes.

Dans les sections franco-allemandes, l'autonomie de l'apprenant est favorisée par le travail dans les centres de documentation et d'information (CDI). Dix établissements en ont été dotés par la France. Ils collaborent entre eux, avec des centres régionaux de documentation pédagogiques (CRDP) en France et avec des institutions homologues en 
Allemagne. Ils offrent aussi leurs services aux autres établissements dans la région ${ }^{10}$. Pour les sections bilingues associant d'autres langues cibles, les institutions culturelles des pays partenaires offrent leur aide.

60 C'est dans « l'usage » de la langue que se manifeste l'apprentissage linguistique. Dans les disciplines spécifiques la langue est « employée »; elle n'est pas l'objet premier du cours, elle en est le véhicule. Ceci a des conséquences sur les réactions de l'enseignant vis-à-vis des fautes linguistiques des élèves. L'enseignant doit encourager l'expression libre de l'apprenant et non sanctionner les fautes.

61 Dans les matières non-linguistiques, la compétence linguistique de l'apprenant est un sous-produit voulu, un produit résiduel mais intentionnel. Le contenu ne doit en aucun cas servir de prétexte à l'enseignement de langue.

62 L'apprentissage de la langue cible est orienté vers la communication réelle, dans des situations concrètes. La formation bilingue exige la pratique authentique de la langue cible avec des locuteurs natifs dans les situations authentiques, par exemple avec des enseignants et des assistants de la culture cible, pendant des échanges scolaires avec intégration dans les familles et dans l'établissement. Les échanges sont un élément constitutif de l'éducation bilingue dès l'âge de onze ans.

63 Enfin, l'éducation bilingue doit être intégrée à la vie scolaire, faire partie du projet d'établissement qui fédère les enseignants et les activités périscolaires.

64 D'autres aspects importants du «modèle allemand» sont évoqués ici pour mémoire, ayant déjà été développés par ailleurs ${ }^{11}$ :

- le terme de « discipline bilingue »;

- le choix des disciplines bilingues ;

- l'éducation bilingue : l'enseignement en langue étrangère et le rôle de la première langue (maternelle); ;

- l'évaluation des résultats obtenus par les élèves ;

- le matériel servant aux cours des disciplines bilingues.

\section{Réussites et échecs}

En 1995, et toujours dans le Land pris comme exemple pour cet article, la Rhénanie-duNord-Westphalie, 11,5\% des Gymnasien offrent une section bilingue, dont $64 \%$ une section anglaise, $25 \%$ une section française et $11 \%$ des sections dans d'autres langues, sections italienne (1), lettone (1), néerlandaise (1), grecque moderne (1), russes (2), espagnole (1).

L'ancienneté des sections varie : l'âge moyen des sections françaises s'élève à douze ans environ, celui des sections anglaises et autres à quatre ans seulement. Les sections anglaises ont connu un essor depuis 1990, tandis que l'accroissement en nombre des sections françaises s'est fait lentement mais régulièrement avec environ une section par an. Pour les autres langues cibles, on constate une saturation ou même, pour les sections russes, une régression - passagère, espérons-le.

67 Les statistiques sur les sections bilingues franco-allemandes montrent que $32,2 \%$ d'élèves quittent la section bilingue à la fin du premier degré. L'étude distingue parmi ces élèves ceux qui la quittent à cause de déménagements $(16,5 \%)$ et ceux qui ont «échoué ", c'està-dire sont passés de la section bilingue à la section normale du Gymnasium ou à un autre type d'établissement moins exigeant (15,7\%). 
68 Au moment du passage du premier au deuxième degré du secondaire (dans le même établissement), un certain nombre d'élèves choisit d'autres priorités que l'éducation bilingue : comparé à l'effectif à l'entrée (classe 5 de la section bilingue), deux tiers environ seulement se retrouveront en classe 11 , qui est la première classe du deuxième degré, pour continuer le cursus bilingue jusqu'à l'Abitur. Il y a très peu d'échecs au deuxième degré des sections bilingues (2,4\%). Mais jusqu'à la fin de la classe 11 (seconde en France) le règlement donne aux élèves une dernière possibilité de se réorienter : $5,6 \%$ des élèves se spécialisent en dehors du cursus bilingue. En somme, 11,2 \% des élèves de la classe 11 quittent encore la section bilingue jusqu'à l'Abitur bilingue où les échecs sont extrêmement rares.

Une étude plus approfondie porte sur les causes de l'échec de 15,7\% des élèves du premier degré. Les professeurs, dans les sections bilingues, indiquent en premier lieu un manque général d'aptitude pour le Gymnasium, en deuxième lieu un manque de concentration, d'application et de persévérance et, loin derrière ces deux causes, un manque d'aptitude linguistique.

70 En aucun cas l'échec en section bilingue ne signifie échec scolaire, mais seulement poursuite des études dans une autre filière ou dans un autre type d'établissement scolaire aboutissant à un diplôme de fin de premier ou deuxième degré.

71 Enfin, un dernier résultat est digne d'être mentionné : la majorité de la population scolaire dans les sections bilingues provient, en effet, de familles qui ont le sens de l'éducation et qui se sont informées des différents types d'établissement et des objectifs et structures des filières à choisir. Mais il est vrai aussi que cette population scolaire est loin d'être représentée par une élite. L'éducation bilingue n'est pas élitiste dans son recrutement, elle accueille les jeunes citoyens de toutes les couches sociales pour former une élite dont notre vieille Europe a besoin.

\section{Perspectives}

72 L'éducation bilingue, selon ce modèle, rayonne sur tous les types d'établissements allemands, sur la Realschule, la Gesamtschule (type d'établissement allemand du secondaire qui ne sélectionne pas les élèves) et même sur quelques Hauptschulen qui, elles, accueillent des élèves qui sont orientés vers un enseignement concret et moins exigeant quant à l'abstraction. Dans les types d'établissements mentionnés, on applique seulement quelques aspects du modèle décrit dans cet article.

73 En parlant de perspectives, il est malheureusement nécessaire de mentionner que tous les Länder, bien que très favorables au principe de l'éducation bilingue, croient nécessaire de limiter l'expansion des sections bilingues - faute d'argent, surtout à la suite de la réunification ${ }^{12}$. En effet, une section bilingue coûte de l'argent. Mais, vu la somme minime nécessaire pour faire fonctionner une section bilingue, il est difficile de comprendre la politique restrictive des responsables. Une section bilingue de la variante intégrative exige de la part de l'instance qui gère les enseignants, le Land fédéral, six périodes par semaine en plus pour le cursus complet de la classe 5 jusqu'à l'Abitur. L'autorité de tutelle, qui est le plus souvent la municipalité, prend en charge le transport scolaire de quelques élèves qui pourraient fréquenter un établissement scolaire plus proche de leur domicile s'ils n'avaient préféré la section bilingue. La collectivité de rattachement met aussi à la disposition des élèves des jeux d'atlas et de manuels des disciplines bilingues dans la 
langue cible. Ces matériels restent dans le stock de l'établissement pour être loués aux élèves. « Le programme bilingue allemand est efficace, et il n'est pas cher ", tel est l'avis $\mathrm{du}$ responsable des sections bilingues au ministère de la culture à Düsseldorf ${ }^{13}$. Ceci témoigne du soutien dont l'éducation bilingue jouit en dépit de la pénurie d'argent actuelle en Allemagne.

Le cursus des sections bilingues en Allemagne a servi aussi à une expérimentation scolaire couronnée de succès en 1994 : la délivrance simultanée (DS) de l'Abitur et du baccalauréat ${ }^{14}$. Pour ce diplôme, des programmes allemands et français du second degré ont été harmonisés. Après les trois établissements pilotes des deux côtés, il y en aura trois autres en France et en Allemagne qui orienteront une partie de leurs élèves de la classe 11 et de la seconde vers la DS à partir de l'année scolaire 1995/1996.

Après environ deux décennies où la formation continue des enseignants des sections bilingues était presque uniquement à la charge des intéressés, les institutions d'État ont commencé à s'engager avec efficacité.

Quant à la formation initiale des futurs enseignants des disciplines bilingues, le Land de Rhénanie-Palatinat a été le premier à esquisser un programme provisoire. Le Land de Rhénanie-du-Nord-Westphalie présentera un programme pour la «classe » de Referendare (enseignant en stage dans le Studienseminar/IUVM) en décembre 1995. Le programme comportera une formation spécifique pour l'enseignement des disciplines bilingues, y compris un stage dans un établissement scolaire du pays partenaire qui implique des contacts avec par exemple des instituts universitaires de formation des maîtres (IUFM) en France. Parallèlement, l'institut de la didactique des langues de l'université de Wuppertal, en Rhénanie-du-Nord-Westphalie, est en train de développer un cursus universitaire visant la didactique de l'enseignement des disciplines bilingues. Les responsables sont convaincus que, dès 1996, une coopération fonctionnelle sera établie, d'une part, entre les formations initiales de l'enseignement supérieur et de l'institut pédagogique (Studienseminar) et, d'autre part, avec la formation continue qui accomplit un travail constant et efficace depuis 1975. La coopération est aussi prévue avec les universités de Besançon et de Lyon 2 ainsi que l'IUFM de Lyon. Les futurs enseignants dans les sections bilingues en Allemagne en profiteront aussi bien que leurs collègues des sections européennes en France.

En plus, un réseau international de recherches et de développement a été institué dans le contexte du Conseil de l'Europe. Il facilitera la coopération et la formation mutuelle par exemple des sections bilingues franco-allemandes en Allemagne et des sections européennes en France. La coopération pourra être très fructueuse car les systèmes français et allemand auront l'essentiel en commun, selon les propos du directeur de la direction des affaires générales, internationales et de la coopération (DAGIC) ${ }^{15}$, qui a déclaré le 23 mai 1992, au Colloque de Saint-Germain-en-Laye, à propos des sections européennes à créer : «Quel sera le système et comment l'avons-nous conçu ? Je dois dire qu'il me revient de rendre ici hommage au système qui nous a inspirés et qui est celui de l'Allemagne. Nous sommes partis de ce qui a été mis en place depuis pas mal d'années en Allemagne, des sections bilingues - dont on sait que les résultats pédagogiques sont excellents, et qui sont un système à la fois élaboré, donnant de bons résultats, mais adapté à un public qui n'est pas un public international mais un public d'élèves allemands, dans leur majorité ${ }^{16} »$. Le jugement positif de la part d'un représentant du premier partenaire de l'Allemagne en Europe est à même de soutenir les efforts en vue d'une politique d'éducation bilingue commune. 
78 On parle souvent de la dimension européenne dans l'enseignement. Elle est profondément enracinée dans les sections bilingues du «modèle allemand » qui, par la voie du projet d'établissement, s'ouvrent aux cultures réciproques et à un plurilinguisme qui voit loin. Les sections bilingues en Allemagne ont déjà fait de grands pas vers l'Europe. Elles ont besoin de nombreux établissements homologues dans les différents pays européens pour d'abord réunir les forces et finalement arriver à une éducation européenne qui ne sera nullement uniforme mais cohérente.

\section{NOTES}

1. Nando Mäsch, «Les disciplines non linguistiques du cursus bilingue », dans: Études de linguistique appliquée, ${ }^{\circ} 96$, octobre-décembre 1994, points 5 et 6

2. Nando Mäsch, «Les disciplines non-linguistiques du cursus bilingue », loc. cit., p. 87 et 88

3. Pour plus de détails: Nando Mäsch, «La recherche d'efficacité - Le cas de la RhénanieWestphalie ", dans : Revue internationale d'éducation de Sèvres, $n^{\circ} 4$, décembre 1994, p. 35-46.

4. Arrêté $n^{\circ} 4.68 / 88$ du 7 décembre 1988

5. À prendre dans le sens de matière à enseigner et non de sanction.

6. Nando Mäsch, «Sachunterricht in der Fremdsprache an Gymnasien mit deutsch-französisch bilingualem Zug », dans : Neusprachliche Mitteilungen, $\mathrm{n}^{\circ}$ 1, 1981, p. 18-28

7. Gerhard Hoffmann, «Bilingualer Unterricht in Rheinland-Pfalz », dans : Mitteilungsblatt des Fachverbandes moderne Fremdsprachen (FMF) des Landesverbandes Rheinland-Pfalz, 1/3/1993, p. 13-17, et Françoise Sauer, Irene Heister, «Bilingualer Unterricht in Rheinland-Pfalz», dans : Der fremdsprachliche Unterricht, Französisch, Heft 9, février 1993, p. 17-23

8. À paraître en 1995-1996, dans la même série du Kultusminister que les Empfehlungen (recommandations) en vigueur.

9. Le programme obligatoire demande pour le premier semestre de la classe 9 de traiter Europa als Traditionsraum, les séquences étant structurées comme section thématique " longitudinale ».

10. Wolfgang Schirmeisen, «CDI... und kein Ende», dans : Schulverwaltung, mars 1995, p. 93-94, et: Martine Boyer-Weimann, Bastianelli, Lowy, Gautherot, «Des CDI à la française en Allemagne ", dans : ARGOS, Revue des B.C.D. \& C.D.I., n 11 (En Europe et ailleurs), Réseau CRDP académie de Créteil, octobre 1993, p. 69-70.

11. Nando Mäsch, « Les disciplines non linguistiques du cursus bilingue », loc. cit.

12. Nando Mäsch, « La recherche de l'efficacité », loc. cit.

13. Ingeborg Christ, « Organisation générale et leçons des expériences allemandes », dans : Études de linguistique appliquées, $\mathrm{n}^{\circ} 96$ (octobre-décembre 1994), p. 73.

14. Friedhelm Dilk, «Un établissement en voie de développement vers l'Europe : le projet de la délivrance simultanée de l'Abitur et du baccalauréat au lycée Friedrich Ebert de Bonn ", dans : Études de linguistique appliquées, $\mathrm{n}^{\circ} 96$ (octobre-décembre 1994), p. 79-88.

15. Direction des affaires générales, internationales et de la coopération au ministère de l'éducation nationale, en France.

16. Anne-Marie Leroy, «Discours de clôture », dans : Actes 92 sur l'enseignement international, lycée international de St-Germain-en-Laye, 1992, p. 32. 
INDEX

Index géographique : Allemagne

Mots-clés : enseignement bilingue, section bilingue, éducation bilingue

\section{AUTEUR}

NANDO MÄSCH

Inspecteur, directeur de l'administration scolaire, Cologne, Allemagne 